13.4

\title{
Использование импульсов микроволнового сверхизлучения для высокоградиентного ускорения электронов в цилиндрическом волноводе с диэлектрической вставкой
}

\author{
(С) Ю.Ю. Данилов ${ }^{1}$, Н.С. Гинзбург ${ }^{1,}$, И.В. Зотова ${ }^{1}$, М.И. Яландин ${ }^{2}$ \\ ${ }^{1}$ Институт прикладной фризики РАН, Нижний Новгород, Россия \\ ${ }^{2}$ Институт электросиизки УрО РАН, Екатеринбург, Россия \\ ๑ E-mail: ginzburg@ipfran.ru
}

Поступило в Редакцию 14 июля 2021 r.

В окончательной редакции 14 июля 2021 г.

Принято к публикации 26 августа 2021 г.

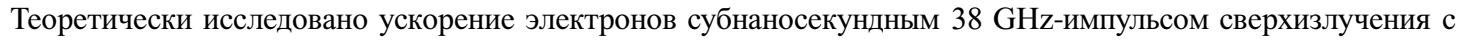
пиковой мощностью $3 \mathrm{GW}$ в цилиндрическом волноводе с трубчатой диэлектрической вставкой. Показано, что прирост энергии и ускоряющий градиент зависят от величины диэлектрической проницаемости вставки. При этом прирост энергии уменьшается, а градиент увеличивается с увеличением диэлектрической проницаемости. Для электрона с начальной энергией $300 \mathrm{keV}$ максимальный ускоряющий градиент достигает $120 \mathrm{MV} / \mathrm{m}$ при приросте энергии $7.2 \mathrm{MeV}$ в волноводе с полистироловой вставкой $(\varepsilon=2.53)$ и $220 \mathrm{MV} / \mathrm{m}$ при приросте энергии $4.5 \mathrm{MeV}$ в волноводе с кварцевой вставкой $(\varepsilon=3.81)$.

Ключевые слова: импульс сверхизлучения, релятивистский электрон, высокоградиентное ускорение, волновод с диэлектрической вставкой, миллиметровый диапазон волн.

DOI: 10.21883/PJTF.2021.23.51780.18962

В стандартных ускоряющих СВЧ-структурах, запитываемых длинноволновыми $(1-10 \mathrm{GHz})$ клистронами, рабочий градиент составляет 30-50 MV/m, а рекордные значения не превышают $100 \mathrm{MV} / \mathrm{m}$. По этой причине для ускорения электронов до высоких энергий приходится создавать длинные и дорогостоящие сооружения. Соответственно повышение темпа ускорения свыше указанного уровня является актуальной задачей в физике ускорителей. При этом, несмотря на возникновение целого ряда новых концепций (включая, например, лазерно-плазменное ускорение и т.п.), актуальными остаются исследования ускорения электронов в полых металлических и диэлектрических структурах, запитываемых СВЧ-излучением [1-6]. При этом повышение градиента ускорения может быть достигнуто при одновременном повышении частоты рабочих полей и уменьшении времени экспозиции структур сильным полем, что позволяет снизить вероятность развития СВЧ-пробоев, являющихся основным ограничивающим фактором. Таким образом, для решения указанной задачи требуются источники мощных ультракоротких электромагнитных импульсов.

В настоящее время наиболее мощные субнаносекундные импульсы в миллиметровом диапазоне реализуются на основе использования эффектов черенковского сверхизлучения (СИ). Генераторы СИ обеспечивают рекордные уровни мощности вплоть до $3 \mathrm{GW}$ в длинноволновой части миллиметрового диапазона $[7,8]$ и до $150 \mathrm{MW}$ в коротковолновой части $[9,10]$. При этом на частоте $38 \mathrm{GHz}$ продемонстрирована возможность когерентного сложения синфазных импульсов СИ, поступающих из нескольких параллельных каналов [11]. В связи с этим представляется привлекательным использование генераторов СИ для тестирования новых схем высокоградиентного ускорения.

В настоящей работе анализируется возможность использования импульсов СИ для тестирования ускорения электронов в канале в виде полого цилиндрического металлического волновода с длиной $L$ и радиусом $R_{2} \mathrm{c}$ трубчатой диэлектрической вставкой с диэлектрической проницаемостью $\varepsilon$ и внутренним радиусом $R_{1}$. Подобная схема ускорения экспериментально исследована в работax $[5,6]$, где в качестве ускоряющего использовался короткий моноимпульс, получаемый выпрямлением лазерного излучения в электрооптическом кристалле [12,13]. Однако такой импульс является полупериодным и испытывает сильное дисперсионное расплывание при распространении в ускоряющем волноводе, что в свою очередь ограничивает абсолютный прирост энергии ускоренных электронов. Преимуществом импульса СИ, содержащего от 5 до 15 периодов заполнения при напряженности ускоряющих полей, сопоставимой с [13], заключается в его относительно узком спектре и соответственно незначительном расплывании на длине ускорительной секции. Дополнительным преимуществом генераторов СИ является достаточно высокий КПД (десятки процентов) по сравнению с крайне низкой эффективностью оптико-терагерцевого преобразования (доли процента в лучших на данный момент экспериментах).

Исследуем ускорение одиночного электрона импульсом СИ, возбуждающим моду $E_{01}$ цилиндрического волновода с диэлектрической вставкой. Будем предпо- 
лагать, что электрон движется прямолинейно вдоль оси волновода. Ускоряющее поле задается распределением по оси волновода $z$ амплитуды продольной компоненты электрического поля указанной моды:

$$
E=\operatorname{Re}\left\{E_{z}(z, t) \exp [i \psi(z, t)]\right\},
$$

где

$$
\psi(z, t)=2 \pi f_{0}\left[\int_{0}^{z} \frac{d z^{\prime}}{v_{e}\left(z^{\prime}\right)}+t_{d}\right]-h z
$$

- фаза электрона в поле ускоряющего импульса СИ с центральной частотой $f_{0} ; v_{e}-$ скорость электрона; $t_{d}$ - время запаздывания инжекции электрона в область взаимодействия относительно пика импульса. Энергия ускоренного электрона $W$ и ускоряющий градиент $G$ определяются соотношениями

$$
\begin{gathered}
W=W_{0}+|e| \operatorname{Re}\left\{\int_{0}^{L} E_{z}(z, t) \exp [i \psi(z, t)] d z\right\}, \\
G=\frac{1}{|e|} \frac{d W}{d z} .
\end{gathered}
$$

Указанные величины оцениваются на основе оптимизации параметров ускорительного канала и времени запаздывания электрона $t_{d}$ на входе волновода. Омические потери в ускорительном канале считаются пренебрежимо малыми. Оптимизация, которая проводилась посредством кода CST Microwave Studio [14], в основном сводится к поиску компромисса между значением амплитуды ускоряющего поля и приемлемым для ускорения дисперсионным расплыванием импульса. Начальная энергия электрона выбиралась равной $300 \mathrm{keV}$, а параметры ускоряющего импульса СИ были близки к описанным в эксперименте [8]. Огибающая импульса с пиковой мощностью $3 \mathrm{GW}$, центральной частотой $38 \mathrm{GHz}$ и длительностью 200 ps по половинному уровню мощности аппроксимировалась гауссовой функцией.

Как следует из рис. 1, $a$, максимальная энергия ускоренного электрона уменьшается, а максимальный ускоряющий градиент увеличивается с увеличением диэлектрической проницаемости вставки $\varepsilon$. Совместное достижение максимальных значений энергии электрона и ускоряющего градиента является невозможным, но для некоторых диэлектриков, например полистирола $(\varepsilon=2.53)$ и кварца $(\varepsilon=3.81)$, возможен приемлемый компромисс. Оптимальные длины $L_{W}$ и $L_{G}$, при которых достигаются максимальный прирост энергии электрона и максимальный ускоряющий градиент, уменьшаются с увеличением диэлектрической проницаемости вставки (рис. 1,b).

При выбранных параметрах ускоряющего импульса оптимальные значения радиусов ускорительного канала и времени запаздывания электрона составляют $R_{2}=3.4 \mathrm{~mm}, R_{1}=0.95 \mathrm{~mm}, t_{d}=253 \mathrm{ps}$ для волновода
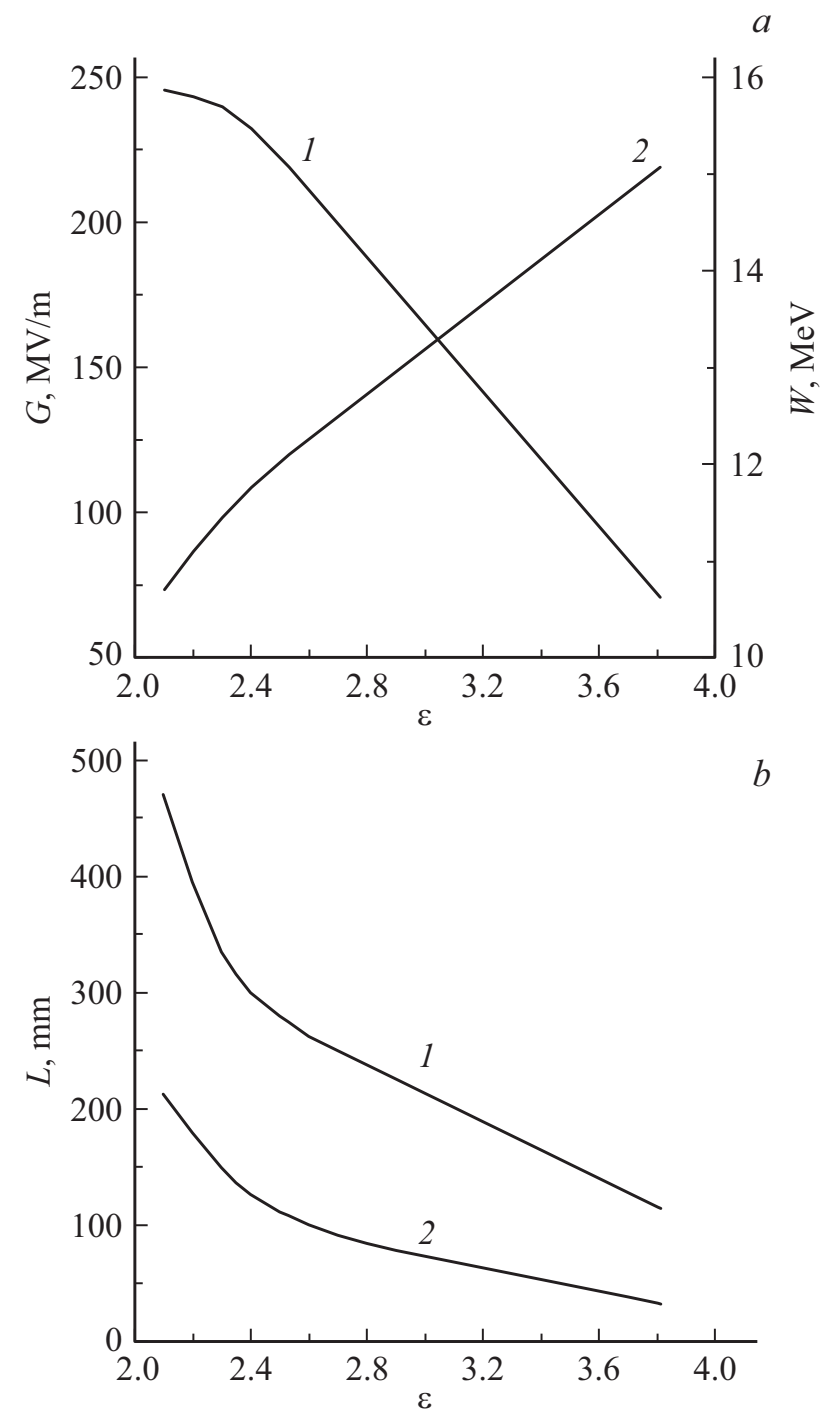

Рис. 1. $a-$ энергия ускоренного электрона W (1) и ускоряющий градиент $G(2) ; b-$ оптимальные длины ускоряющего волновода, обеспечивающие максимальное значение прироста энергии $L_{W}(1)$ и темпа ускорения $L_{G}(2)$, как функции диэлектрической проницаемости вставки $\varepsilon$.

со вставкой из полистирола и $R_{2}=2.45 \mathrm{~mm}, R_{1}=1 \mathrm{~mm}$, $t_{d}=200 \mathrm{ps}$ для волновода со вставкой из кварца. Зависимости энергии ускоренного электрона $W$ ускоряющего градиента $G$ от нормированной длины волновода $\xi=z / L$ для каждого набора оптимальных параметров представлены на рис. 2, $a$ и $b$. Ускорение электрона $3 \mathrm{GW}$-импульсом СИ позволяет достичь прироста энергии электрона $14.8 \mathrm{MeV}$ на длине $275 \mathrm{~mm}$ в волноводе со вставкой из полистирола и $10.3 \mathrm{MeV}$ на длине $115 \mathrm{~mm}$ в волноводе со вставкой из кварца. В свою очередь максимальный ускоряющий градиент составляет $120 \mathrm{MV} / \mathrm{m}$ при приросте энергии электрона $7.2 \mathrm{MeV}$ на длине $109 \mathrm{~mm}$ в волноводе с полистиролом и $220 \mathrm{MV} / \mathrm{m}$ при приросте энергии электрона $4.5 \mathrm{MeV}$ на длине $33 \mathrm{~mm}$ в волноводе с кварцем. 

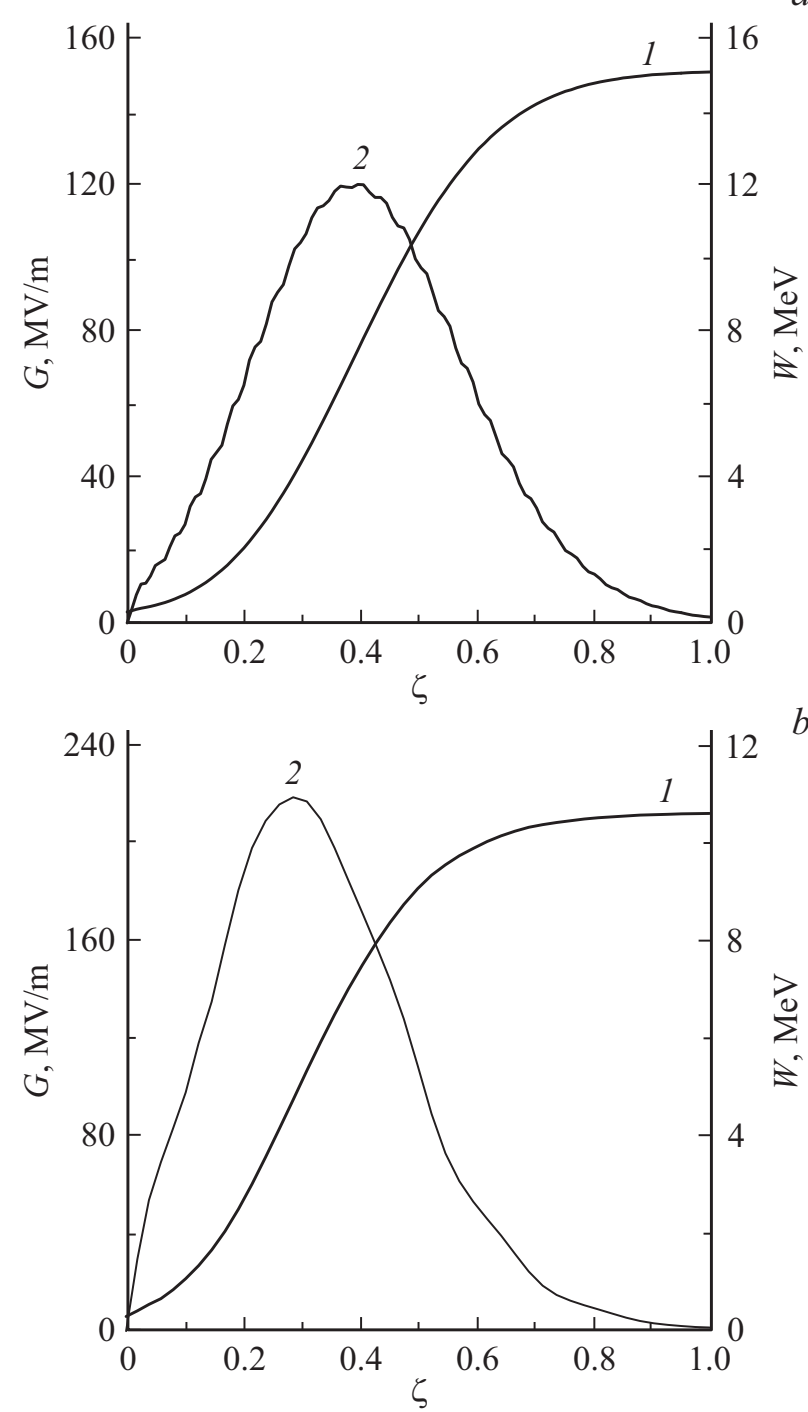

Рис. 2. Энергия ускоренного электрона $W(1)$ и ускоряющий градиент $G(2)$ как функции нормированной длины волновода $\xi$. $a$ - для вставки из полистирола $(\varepsilon=2.53), b$ - для вставки из кварца $(\varepsilon=3.81)$.

Импульс СИ является относительно узкополосным, и его дисперсионное расплывание на обозначенных длинах незначительно. Поэтому энергия ускоренного электрона и ускоряющий градиент представляют собой квазипериодические функции времени запаздывания электрона вне зависимости от выбора диэлектрической вставки (рис. $3, a, b)$. Таким образом, селекция времени запаздывания позволяет минимизировать влияние отклонений параметров ускорительного канала от оптимальных значений.

При тестировании описанного метода ускорения в модельном эксперименте при пониженном уровне мощности импульсов СИ предполагается использовать экспериментальный стенд, состоящий из двух ускорителей RADAN [15], синхронизированных с субнаносекундной точностью [16]. Один из ускорителей генерирует импульс СИ, который через линию передачи поступает в ускорительный канал, где движется электронный пучок, инжектируемый вторым ускорителем. В дальнейшем для выхода на расчетные параметры возможно использовать когерентное сложение (с помощью волноводных сумматоров мощности) синфазных импульсов СИ, генерируемых в нескольких жестко синхронизированных каналах (см. [11]).

Заметим в заключение, что в коротковолновой части миллиметрового диапазона те же максимальные значения ускоряющего градиента могут быть достигнуты при ускорении электрона импульсом СИ со значительно меньшей пиковой мощностью 220 (150) MW на частоте 140 (170) GHz. При этом ускоряющий волновод
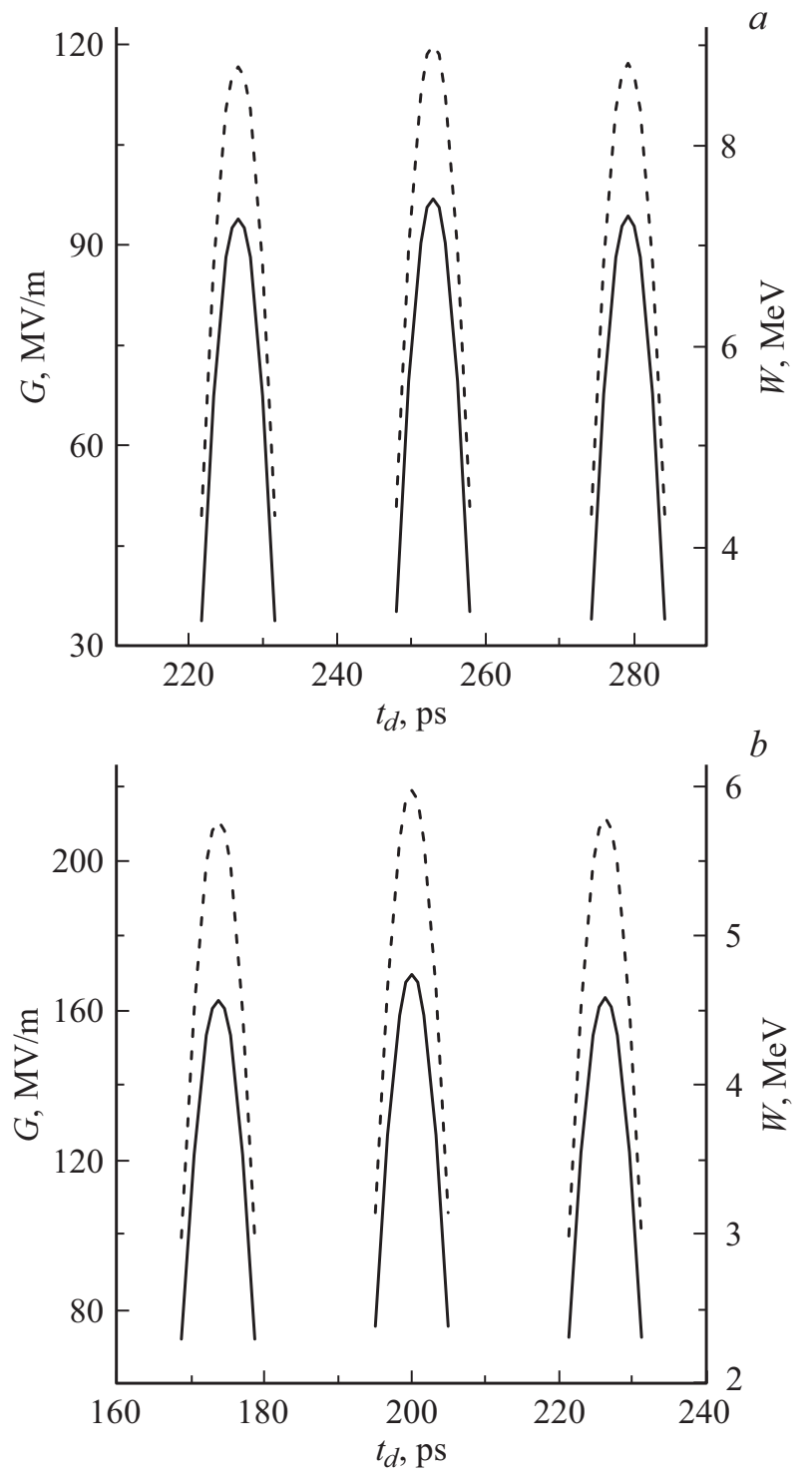

Рис. 3. Энергия ускоренного электрона $W$ (сплошная линия) и ускоряющий градиент $G$ (штриховая линия) как функции времени запаздывания электрона $t_{d}$ для волновода длиной $L_{G}$ с различными вставками. $a-$ вставка из полистирола $(\varepsilon=2.53), b-$ вставка из кварца $(\varepsilon=3.81)$. 
становится более компактным, но энергия ускоренного электрона будет значительно меньше. Длина структуры и прирост энергии электрона составляют 29.5 (24.5) mm и 1.9 (1.6) MeV для волновода со вставкой из полистирола и 9 (7.5) $\mathrm{mm}$ и 1.2 (1) MeV для волновода со вставкой из кварца. В настоящий момент указанные уровни мощности импульсов СИ в коротковолновой части миллиметрового диапазона еще не достигнуты. Однако эксперименты по генерации импульсов СИ при возбуждении поверхностных волн $[9,10]$ указывают на такую возможность.

\section{Финансирование работы}

Работа выполнена при финансовой поддержке Российского фонда фундаментальных исследований (проект № 21-19-00260).

\section{Конфликт интересов}

Авторы заявляют, что у них нет конфликта интересов.

\section{Список литературы}

[1] P. Schoessow, M.E. Conde, W. Gai, R. Konecny, J. Power, J. Simpson, J. Appl. Phys., 84 (2), 663 (1998).

[2] A.M. Cook, M.C. Thompson, S.Y. Toshitsky, G. Travish, O.B. Williams, J.B. Rozenzwheig, Phys. Rev. Lett., 103 (9), 095003 (2009). DOI: 10.1103/PhysRevLett.103.095003

[3] M.A.K. Othman, J. Picard, S. Schaub, V.A. Dolgashev, S.M. Lewis, J. Neilson, A. Haase, S. Jawla, B. Spataro, R.J. Temkin, S. Tantawi, E.A. Nanni, Appl. Phys. Lett., 117 (7), 073502 (2020). DOI: 10.1063/5.0011397

[4] X. Lu, J.F. Picard, M.A. Shapiro, I. Mastovsky, R.J. Temkin, M. Conde, J.G. Power, J. Shao, E.E. Wisniewski, M. Peng, G. Ha, J. Seok, S. Doran, C. Jing, Appl. Phys. Lett., 116 (26), 264102 (2020). DOI: 10.1063/5.0012671

[5] E.A. Nanni, W.R. Huang, K.-H. Hong, K. Ravi, A. Fallahi, F.X. Kärtner, Nature Commun., 6, 8486 (2015). DOI: $10.1038 /$ ncomms 9486

[6] L.J. Wong, A. Fallahi, F.X. Kärtner, Opt. Express., 21 (8), 9792 (2013). DOI: 10.1364/OE.21.009792

[7] V.V. Rostov, I.V. Romanchenko, M.S. Pedos, S.N. Rukin, K.A. Sharypov, V.G. Shpak, S.A. Shunailov, M.R. Ul'masculov, M.I. Yalandin, Phys. Plasmas., 23 (9), 093103 (2016). DOI: $10.1063 / 1.4962189$

[8] В.В. Ростов, А.В. Гунин, И.В. Романченко, М.С. Педос, С.Н. Рукин, К.А. Шарыпов, С.А. Шунайлов, М.Р. Ульмаскулов, М.И. Яландин, Изв. вузов. Физика, 60 (8), 55 (2017).

[9] N.S. Ginzburg, A.M. Malkin, A.S. Sergeev, I.V. Zheleznov, V.Yu. Zaslavsky, I.V. Zotova, G.Sh. Boltachev, K.A. Sharypov, S.A. Shunailov, M.R. Ulmaskulov, M.I. Yalandin, Phys. Rev. Lett., 117 (20), 204081 (2016). DOI: 10.1103/PhysRevLett.117.204801

[10] N.S. Ginzburg, V.Yu. Zaslavsky, A.M. Malkin, A.S. Sergeev, I.V. Zotova, K.A. Sharypov, S.A. Shunailov, V.G. Shpak, M.R. Ul'masculov, M.I. Yalandin, Appl. Phys. Lett., 117 (18), 183505 (2020). DOI: 10.1063/5.0026814
[11] N.S. Ginzburg, A.W. Cross, A.A. Golovanov, G.A. Mesyats, M.S. Pedos, A.D.R. Phelps, I.V. Romanchenko, V.V. Rostov, S.N. Rukin, K.A. Sharypov, V.G. Shpak, S.A. Shunailov, M.R. Ulmaskulov, M.I. Yalandin, I.V. Zotova, Phys. Rev. Lett., 115 (17), 114802 (2015). DOI: $10.1103 /$ PhysRevLett.115.114802

[12] C. Vicario, A.V. Ovchinnokov, S.I. Ashtikov, M.B. Agranat, V.E. Fortov, C.P. Hauri, Opt. Lett., 39 (23), 6632 (2014). DOI: $10.1364 /$ OL.39.006632

[13] H. Hirori, A. Doi, F. Blanchard, K. Tanaka, Appl. Phys. Lett., 98 (9), 091106 (2011). DOI: 10.1063/1.3560062

[14] CST Microwave Studio (2020) [Электронный pecypc]. URL: http://www.cst.com

[15] В.Г. Шпак, С.А. Шунайлов, М.И. Яландин, А.Н. Дядьков, Приборы и техника эксперимента, № 1, 149 (1993).

[16] А.Г. Реутова, М.Р. Ульмаскулов, А.К. Шарыпов, В.Г. Шпак, С.А. Шунайлов, М.И. Яландин, В.И. Белоусов, Н.С. Гинзбург, Г.Г. Денисов, И.В. Зотова, Р.М. Розенталь, А.С. Сергеев, Письма в ЖЭТФ, 82 (5), 295 (2005). 